\title{
Bioinformatics analysis reveals different gene expression patterns in the annulus fibrosis and nucleus pulpous during intervertebral disc degeneration
}

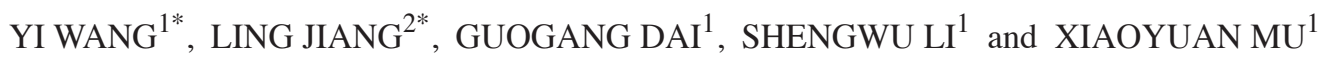 \\ ${ }^{1}$ Cervicodynia/Omalgia/Lumbago/Sciatica Department 2, Sichuan Provincial Orthopedic Hospital, Chengdu, \\ Sichuan 610041; ${ }^{2}$ College Hospital, Sichuan Agricultural University, Chengdu, Sichuan 611130, P.R. China
}

Received June 5, 2018; Accepted September 21, 2018

DOI: $10.3892 /$ etm.2018.6884

\begin{abstract}
Degeneration of the intervertebral disc (IVD), which consists of the annulus fibrosus (AF) and nucleus pulposus (NP), is a multifactorial physiological process associated with lower back pain. Despite decades of research, the knowledge of the underlying molecular mechanisms of IVD degeneration (IDD) has remained limited. The present study aimed to reveal the differential gene expression patterns in AF and NP during the process of IDD and to identify key biomarkers contributing to these differences. The microarray dataset GSE70362 containing $24 \mathrm{AF}$ and $24 \mathrm{NP}$ samples was retrieved from the Gene Expression Omnibus database. Of these, 8 healthy samples were discarded. GeneSpring11.5 software was employed to identify differentially expressed genes (DEGs). Metascape online tools were used to perform enrichment analyses. Finally, the DEGs were mapped with the Search Tool for the Retrieval of Interacting Genes, and a protein-protein interaction (PPI) network was constructed in Cytoscape software. A total of 87 DEGs were identified. Gene ontology enrichment revealed that these DEGs were mainly involved in the inflammatory response, the extracellular matrix and RNA polymerase II transcription factor activity. Pathway enrichment revealed that the DEGs were mainly involved in the transforming growth
\end{abstract}

Correspondence to: $\mathrm{Dr} \mathrm{Yi}$ Wang, Cervicodynia/Omalgia/ Lumbago/Sciatica Department 2, Sichuan Provincial Orthopedic Hospital, 132 West First Section First Ring Road, Chengdu, Sichuan 610041, P.R. China

E-mail: yiwang1984@hotmail.com

*Contributed equally

Abbreviations: IVD, intervertebral disc; IDD, IVD degeneration; PPI, protein-protein interaction; $\mathrm{AF}$, annulus fibrosus; NP, nucleus pulposus; DEG, differentially expressed gene; ECM, extracellular matrix; BP, biological process; MF, molecular function; $\mathrm{CC}$, cellular component; KEGG, Kyoto Encyclopedia of Genes and Genomes

Key words: differential expression, enrichment analysis, protein-protein interaction network factor (TGF- $\beta$ ) and estrogen signaling pathways. Matrix metalloproteinase (MMP)1 and interleukin (IL)6 were included in the genes enriched in rheumatoid arthritis, whereas bone morphogenetic protein (BMP)2 and thrombospondin 1 (THBS1) were among the genes enriched in the TGF- $\beta$ signaling pathway. In the PPI network, IL6 was identified as the central gene. In conclusion, as MMP1 has been demonstrated degrade collagen III at higher rates compared with other types of collagen (which is at a higher quantity in AF than NP), collagen types may be in different distribution patterns, which may contribute to the upregulation of MMP1 in AF. Differences in the expression of BMP2, ESR1 and THBS1 may explain for the pathological differences between AF and NP. IL6 may have a key role in different degeneration processes in AF and NP.

\section{Introduction}

Intervertebral disc (IVD) degeneration (IDD) is considered a primary contributor to disc degeneration disease and is associated with lower back pain (1), which has become a serious public health issue and causes an enormous economic burden (2-5). Despite its significant clinical importance and prevalence, and its extensive impact on general health, current clinical management techniques, which include standard or endoscopic lumbar discectomy and intradiscal thermal annuloplasty, are associated with a substantial amount of recurrence (6-9). In addition, lumbar fusion increases the risk of adjacent segment degeneration (10).

Comprehensive knowledge of the mechanisms underlying IDD is critical for the development of successful therapeutic strategies. Over decades of research, increasing attention has been paid to the molecular mechanisms underlying IDD. Notable improvements in the knowledge of the pathological processes have been achieved (11), with multifactorial bioprocesses proven to contribute to IDD (12).

However, an enormous number of different molecules that interact with each other are associated with IDD, indicating that the pathological process is highly complex. In the abovementioned previous studies, only a small number of molecular factors have been investigated via classic experimental approaches, and thus, the current understanding of the molecular mechanisms involved in the initiation and 
progression of IDD remains limited. Therefore, clinical strategies remain limited, and the development of novel therapeutics depends on acquiring further insight into the molecular basis and pathophysiology underlying IDD.

The IVD is a complex structure, comprising fibrocartilaginous annulus fibrosis (AF) on the outside and highly gelatinous nucleus pulposus (NP) on the inside. The biochemical characteristics of IDD include extracellular matrix (ECM) degradation $(13,14)$. The ECM of IVDs consists primarily of collagen type III in the inner AF, type I in the outer AF and type II in the NP (15-17). The biological structure differs between AF and NP, and this difference was hypothesized to lead to different pathological processes in tissues from these two regions of the IVD during degeneration. The present study assessed possible differences in gene expression between the $\mathrm{AF}$ and NP in IDD by performing a bioinformatics analysis of gene expression profiles of $\mathrm{AF}$ and $\mathrm{NP}$ from degenerated IVDs.

\section{Materials and methods}

Acquisition of expression data. The primary dataset GSE70362 was downloaded from the Gene Expression Omnibus (GEO) database (http://www.ncbi.nlm.nih.gov/geo). The GSE70362 dataset, which is based on the Affymetrix GPL17810 platform (HG-U133_Plus_2; Affymetrix; Thermo Fisher Scientific Inc., Waltham, MA, USA), was uploaded by Kazezian et al (18) in 2015. It contains the gene expression data for 48 IVD tissues, including $24 \mathrm{AF}$ and $24 \mathrm{NP}$ paired samples. The original signal intensity data in CEL format files (annotation edition information), matrix files and classifications based on the Thompson grading system (19) were downloaded. A total of 8 samples (GSM1725800, -1725801, -1725810, -1725811, $-1725814,-1725815,-1725822$ and -1725823$)$ were discarded, as all of them were classified as Thompson grade I and were considered healthy. The remaining 40 samples were classified as Thompson grade I-II to $\mathrm{V}$ and consisted of $20 \mathrm{AF}$ samples and $20 \mathrm{NP}$ samples. To annotate the data, the original probe IDs were transformed into Gene symbol and Entrez IDs. Any probes that were not mapped to any GeneID or that were not identified were discarded.

Identification of differentially expressed genes (DEGs). A significance analysis of the microarray data was performed by using GeneSpring GX 11.5 software (Agilent Technologies, Inc., Santa Clara, CA, USA) to identify DEGs. After pre-processing the initial data via the Robust Multi-array Average procedure, the probe sets with intensity values between 20 and 100\% were retained. For statistical analysis, an unpaired t-test was performed with a threshold P-value of 0.05 and absolute fold change (FC) of 2, and a Benjamini-Hochberg procedure for multiple testing was applied. Multiexperiment Viewer software (version 4.9.0; http://mev.tm4.org/) was used to construct a heat map of the DEGs identified.

Enrichment analysis. DEGs were analyzed using online tools in Metascape (http://metascape.org/). Functional enrichment was performed in 3 categories of GO terms: Biological process (BP), molecular function (MF) and cellular component (CC). Kyoto Encyclopedia of Genes and Genomes (KEGG) pathway enrichment was also performed. All genes in the genome were used as the enrichment background. Terms with a P-value of $<0.01$, a minimum count of 3 and an enrichment factor of $>1.5$ (the enrichment factor is the ratio of the observed count to the count expected by chance) were collected and grouped into clusters based on their membership similarities. More specifically, P-values were calculated based on the cumulative hypergeometric distribution. Q-values were calculated using the Benjamini-Hochberg procedure to account for multiple testing. Kappa scores were used as the similarity metric when performing hierarchical clustering of the enriched terms; sub-trees with a similarity of $>0.3$ were considered a cluster. The most significant term within a cluster was selected as the one representing the cluster.

Protein-protein interaction (PPI) network. To evaluate potential PPIs, DEGs were mapped using the Search Tool for the Retrieval of Interacting Genes (STRING; https://string-db.org) under the default settings; nodes lacking a connection in the network were excluded. Subsequently, a PPI network with a combined interaction score of $>0.4$ was constructed by using Cytoscape software (version 3.6.3; http://www.cytoscape. org/). DEGs with a degree centrality of $>5.0$ were identified as hub genes by using the plug-in CentiScaPe. The MCODE plug-in was applied to identify significant modules with the following criteria: 'Degree cutoff $=2$ ', 'node score cutoff $=0.2$ ', ' $\mathrm{k}$-core $=2$ ' and ' $\max$ depth $=100$ '.

\section{Results}

DEGs. A total of 1,514 genes were identified to be significantly differently expressed between $\mathrm{AF}$ and NP samples $(\mathrm{P}<0.05)$. Of these, 863 genes were downregulated and 651 genes were upregulated. Further analysis identified 87 genes as DEGs with an absolute FC of $>2$. The DEGs accounted for $0.16 \%$ of the total transcriptome (Fig. 1). Among these 87 DEGs, 49 were upregulated and 38 were downregulated. The DEGs were ranked according to their FC, and the top 10 DEGs are listed in Table I. Fig. 2 presents the hierarchical clustering of the DEGs, with the original data normalized using the z-score to indexes between-3 and 3 .

Based on the GO enrichment, the DEGs were enriched in 221 terms in the category BP, 7 in the category CC and 13 in the category MF. The top $30 \mathrm{GO}$ terms in which the DEGs were enriched according to the gene count are presented in Fig. 3 and Table II. The DEGs were significantly enriched in a total of 8 KEGG pathways as presented in Fig. 4 and Table III.

Gene ontology enrichment revealed that these DEGs were mainly involved in the inflammatory response, the extracellular matrix and RNA polymerase II transcription factor activity. Pathway enrichment revealed that the DEGs were mainly involved in the transforming growth factor (TGF- $\beta$ ) and estrogen signaling pathways. Matrix metalloproteinase (MMP) 1 and interleukin (IL)6 were included in the genes enriched in rheumatoid arthritis, whereas bone morphogenetic protein (BMP)2 and thrombospondin 1 (THBS1) were among the genes enriched in the TGF- $\beta$ signaling pathway.

PPI network and module. As presented in Fig. 5, the PPI network comprised 40 connected nodes and 50 edges, and 
Table I. Top 10 most highly dysregulated genes.

\begin{tabular}{|c|c|c|c|c|}
\hline Gene & $\begin{array}{c}\text { Absolute } \\
\text { FC }\end{array}$ & Regulation & P-value & Q-value \\
\hline SPARCL1 & 5.77 & Up & $1.03 \times 10^{-4}$ & $1.26 \times 10^{-2}$ \\
\hline MMP1 & 5.75 & Up & $1.32 \times 10^{-5}$ & $4.34 \times 10^{-3}$ \\
\hline IBSP & 5.16 & Up & $1.04 \times 10^{-3}$ & $4.35 \times 10^{-2}$ \\
\hline RGS1 & 4.64 & Down & $5.30 \times 10^{-6}$ & $2.44 \times 10^{-3}$ \\
\hline THBS1 & 4.25 & Up & $6.66 \times 10^{-10}$ & $7.12 \times 10^{-6}$ \\
\hline FBLN1 & 4.18 & $\mathrm{Up}$ & $4.32 \times 10^{-8}$ & $1.39 \times 10^{-4}$ \\
\hline EPB41L3 & 3.97 & Down & $6.48 \times 10^{-11}$ & $1.77 \times 10^{-6}$ \\
\hline MEG3 & 3.82 & Up & $1.61 \times 10^{-6}$ & $1.26 \times 10^{-3}$ \\
\hline HSPA6 & 3.65 & Up & $3.16 \times 10^{-4}$ & $2.25 \times 10^{-2}$ \\
\hline IRX2 & 3.40 & Up & $2.02 \times 10^{-10}$ & $2.76 \times 10^{-6}$ \\
\hline
\end{tabular}

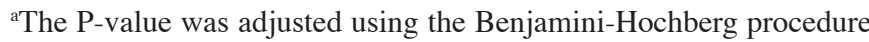
and was denoted as the Q-value. FC, fold change.

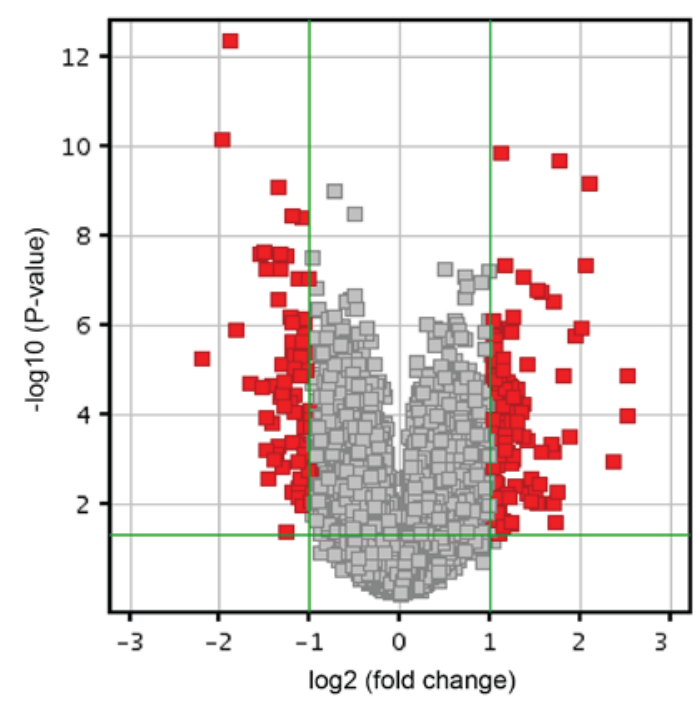

Figure 1. Volcano plot displaying the DEGs. The expression levels of 1,514 genes were significantly different between $\mathrm{AF}$ and $\mathrm{NP}(\mathrm{P}<0.05)$. A total of 87 genes were identified as DEGs ( $\mathrm{P}<0.05$, absolute $\mathrm{FC}>2$; red squares). The P-value was adjusted using the Benjamini-Hochberg procedure. FC, fold change; NP, nucleus pulposus; AF, annulus fibrosus; DEG, differentially expressed gene.

IL6, THBS1, forkhead box (FOX)A2, BMP2, estrogen receptor $(\mathrm{ESR}) 1$, heat shock protein $\mathrm{A} 4$, suppressor of cytokine signaling (SOCS)3 and early growth response 1 were identified as hub genes. One significant module containing 10 nodes and 15 edges was screened out (Fig. 6).

\section{Discussion}

DNA chips allow for the simultaneous detection of the expression of tens of thousands of genes at one time in a single DNA microarray, which facilitates the further identification of DEGs in pathological processes and investigations applying this technology may substantially enhance the knowledge of the molecular mechanisms of IDD. As the DNA microarray has

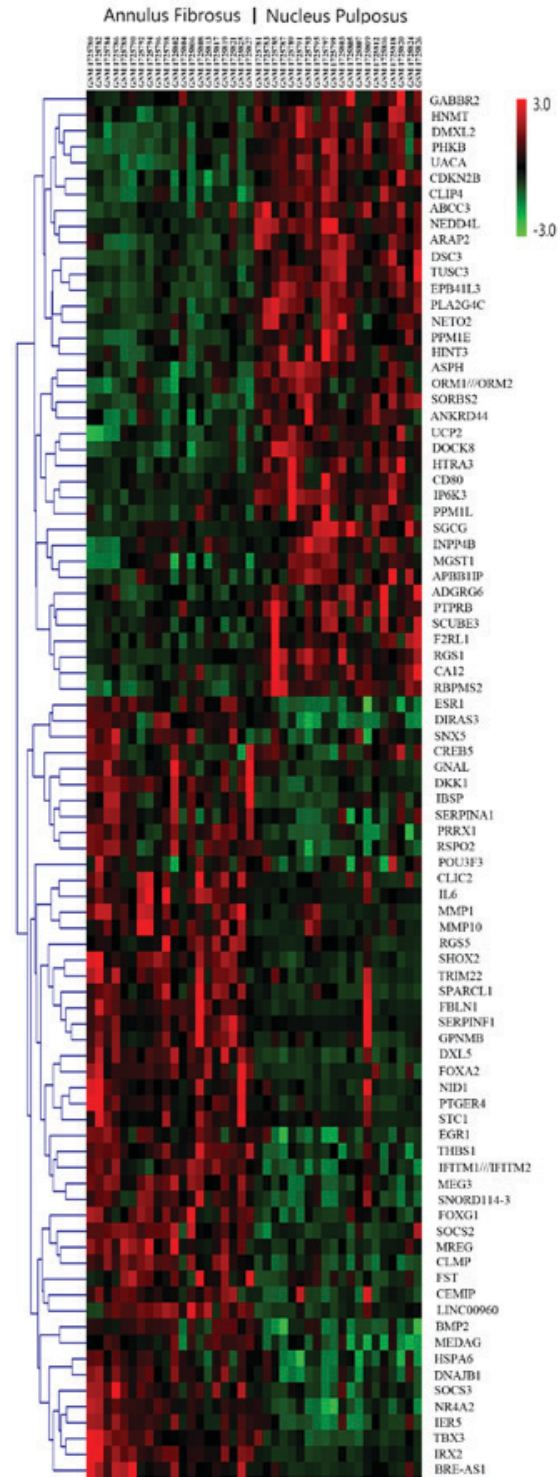

Figure 2. Heatmap displaying hierarchical clustering of the 87 differentially expressed genes in annulus fibrosus and nucleus pulposus (red, upregulated; green, downregulated). The original data were normalized using the z-score to indexes between -3 and 3 .

become popular in recent years, there has been an increased use of transcriptomic approaches in the investigation of the pathophysiology of IDD at the molecular level.

Analysis of the expression spectrum of AF tissues in a previous study revealed that, compared to AF in less degenerated IVDs (Thompson grades I-III), bradykinin receptor B1, calcitonin gene-related peptide and catechol-O-methyltransferase (genes associated with pain), as well as nerve growth factor, were significantly upregulated in more degenerated IVDs (grades IV and V); in addition, numerous genes encoding chemokines and pro-inflammatory cytokines were markedly affected (20). Another microarray analysis indicated differential expression of proteins that bind aspirin in degenerate disc specimens, with higher levels detected in the more degenerated IVDs (grade IV) than in the less degenerated ones (grades I, II and III) (21). Tsai et al (22) reported on 14 DEGs, including periostin, insulin-like growth factor binding protein (IGFB)P6 and 


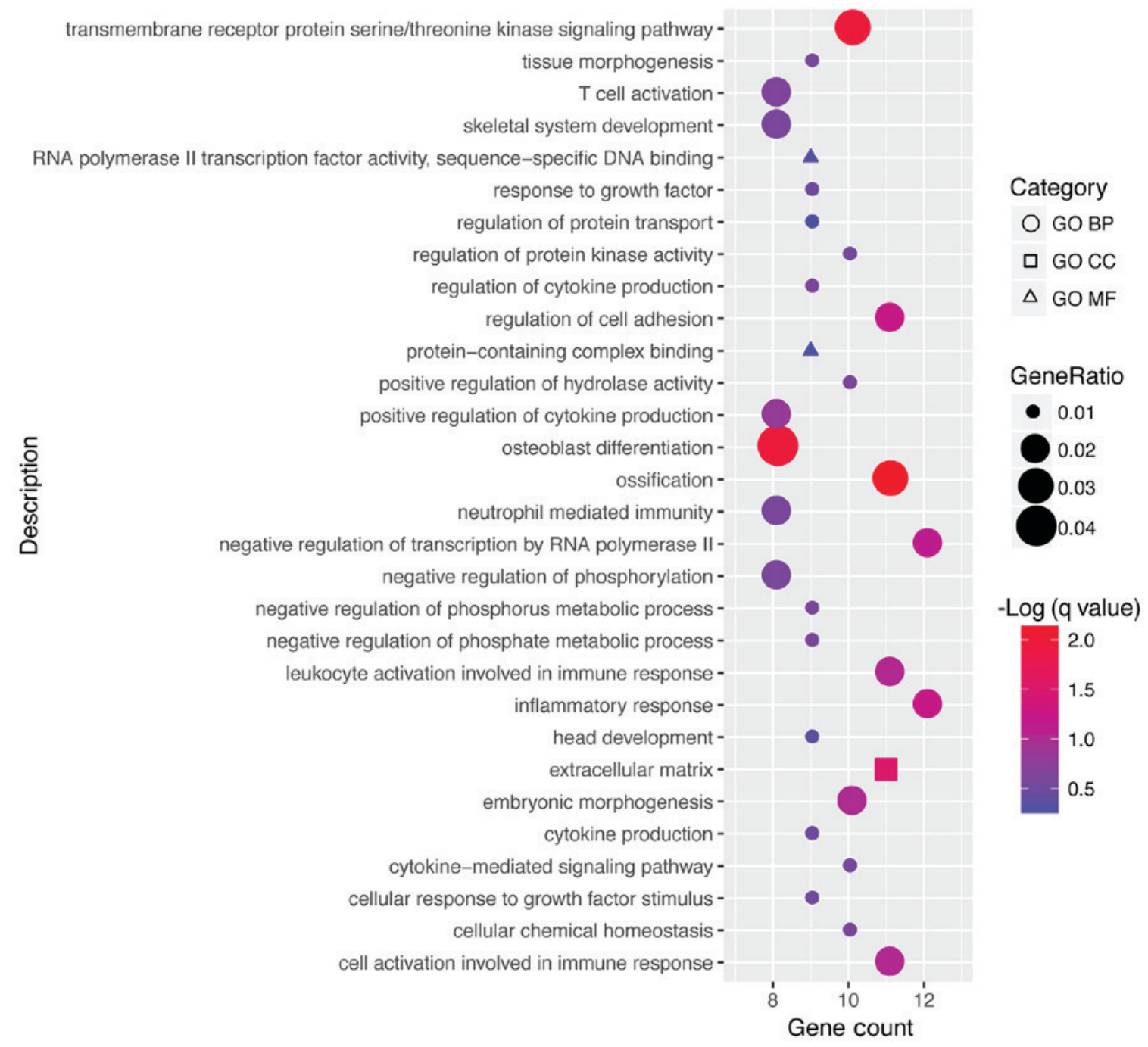

Figure 3. The top 30 ranked GO terms according to gene count. 'Qvalue' is the P-value adjusted using the Benjamini-Hochberg procedure. 'Log (Qvalue)' is the $\log 10$ of the q-value. 'Gene count' is the number of genes enriched in a GO term. 'Gene ratio' is the percentage of total DEGs in the given GO term (only input genes with at least one GO term annotation were included in the calculation). GO, gene ontology; BP, biological process; MF, molecular function; CC, cellular component.

MMP-2, in the NP of degenerated IVDs. Via a genome-wide analysis, Gruber et al (23) identified 424 DEGs in the NP from degenerate IVDs compared with that in normal NP cells and proposed critical genes associated with disc degeneration; these were mainly involved in the ECM, ECM proteolysis, cell proliferation and apoptosis, and also comprised growth factors and inflammatory mediators. He et al (24) compared gene expression data of degenerated IVDs with those of non-degenerated samples and identified 961 DEGs, including 846 DEGs in disc tissues of grade-III IVDs and 1,137 in disc tissues of grade-IV IVDs. They hypothesized that tumor protein 53 has an important role in IDD by impacting neovascularization and infiltration in $\mathrm{AF}$, while ubiquitin $\mathrm{C}$ participated by blocking of cell proliferation in the AF. Kazezian et al (18) obtained microarray data from AF tissues from degenerated and non-degenerated IVDs and identified 238 DEGs in the AF of degenerated IVDs. The dysregulated genes were mainly enriched in the areas of cell proliferation and cellular growth, and were adversely disrupted by IGFBP3 and interferon induced protein with tetratricopeptide repeats 3. Tang et al (25) identified 53 DEGs in degenerated IVDs compared with non-degenerated ones, of which 16 genes were downregulated and 37 were upregulated; bioinformatics analysis revealed that the DEGs were associated with TGF- $\beta$ and the ECM, and MMP2 was indicated to have the highest degree of interaction in the PPI network. Guo et al (26) identified 35 genes that were differentially expressed in AF and NP of degenerated IVDs compared with non-degenerated tissues by analyzing microarray data. Their results suggested that collagen type VI $\alpha 2$ chain, integrin-binding sialoprotein, RAP1A and FOXF2 serve important roles in the focal adhesion signaling pathway associated with IDD and that interferon induced protein with tetratricopeptide repeats $1,-2$ and -3 may accelerate degenerative processes by affecting ECM organization.

The original study that provided the microarray dataset GSE70362 focusing on AF identified 238 dysregulated genes indegenerated AF in comparison with non-degenerated AF (24). These dysregulated genes were enriched in the areas of cellular growth, cell proliferation and inflammatory response, thus confirming that the interferon signaling pathway was significantly dysregulated and activated (24). However, all previous studies employing DNA microarrays to investigate the molecular mechanisms of IDD compared degenerated AF, NP 


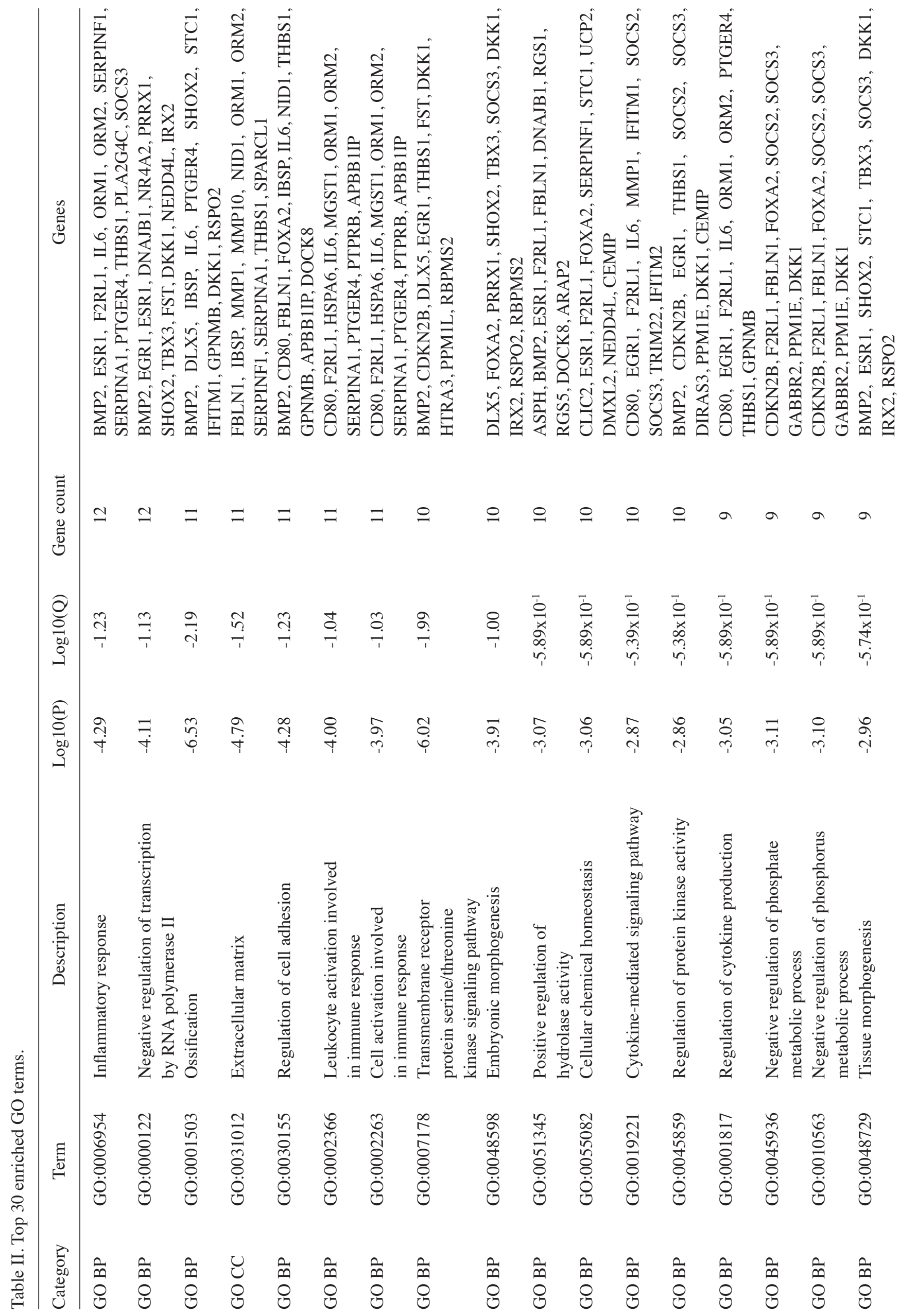




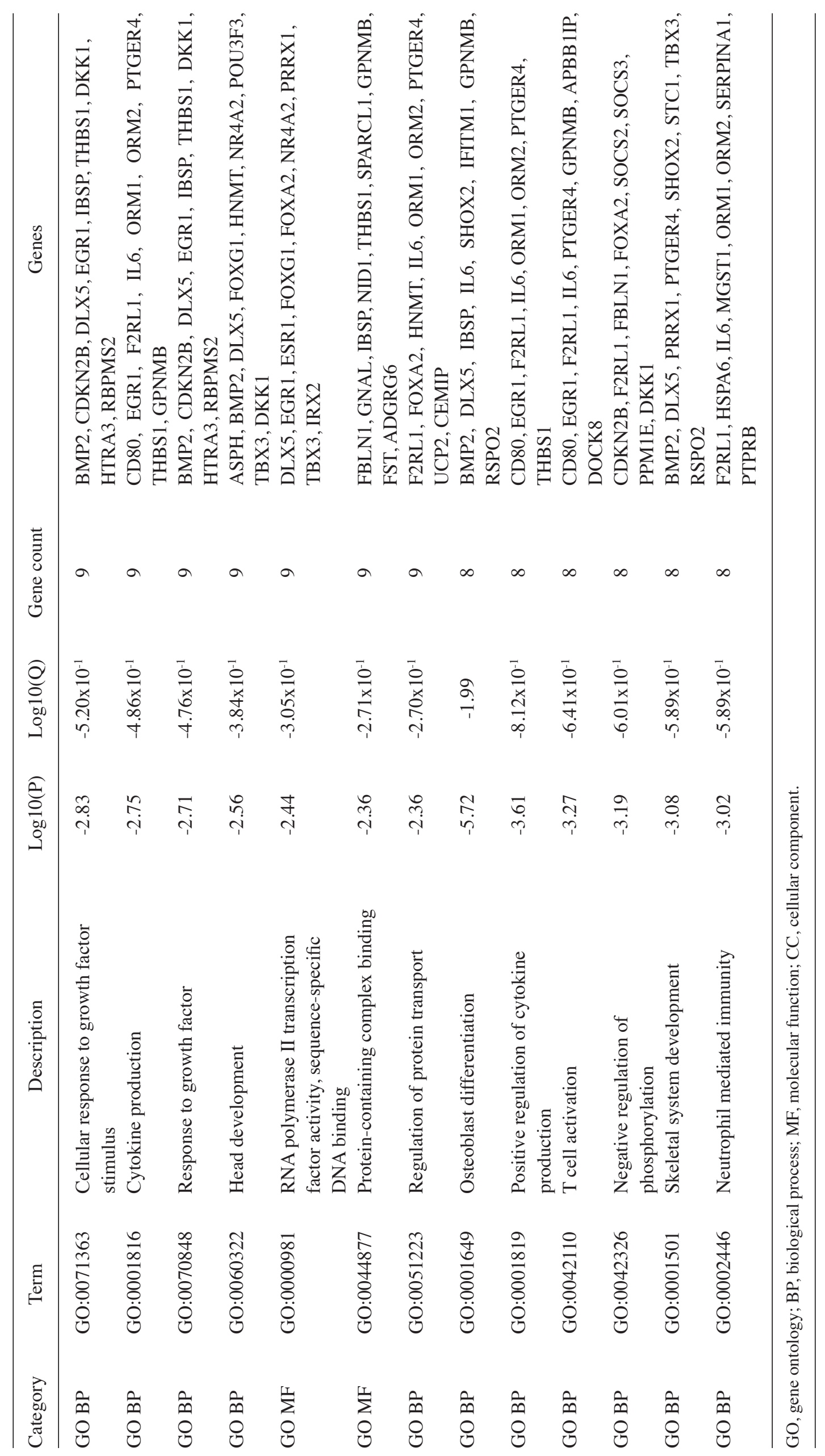


Table III. KEGG pathway enrichment analysis.

\begin{tabular}{lllll}
\hline Term & \multicolumn{1}{c}{ Description } & Log10 $(\mathrm{P})$ & $\log 10(\mathrm{Q})$ & Genes \\
\hline hsa04350 & TGF- $\beta$ signaling pathway & -3.426090165 & -0.780 & BMP2, CDKN2B, THBS1, FST \\
hsa04915 & Estrogen signaling pathway & -3.172403712 & -0.780 & ESR1, HSPA6, GABBR2, CREB5 \\
hsa04917 & Prolactin signaling pathway & -2.542647382 & -0.326 & ESR1, SOCS2, SOCS3 \\
hsa05164 & Influenza A & -2.272816914 & -0.241 & HSPA6, DNAJB1, IL6, SOCS3 \\
hsa04931 & Insulin resistance & -2.029682792 & -0.228 & IL6, SOCS3, CREB5 \\
hsa04668 & TNF signaling pathway & -2.018699722 & -0.228 & IL6, SOCS3, CREB5 \\
hsa05323 & Rheumatoid arthritis & -2.236138588 & -0.241 & CD80, IL6, MMP1 \\
& Inflammatory mediator & & & F2RL1, PTGER4, PLA2G4C \\
hsa04750 & Regulation of TRP channels & -2.146268906 & -0.231 & .
\end{tabular}

The Q-value is the P-value adjusted using the Benjamini-Hochberg procedure. hsa, Homo sapiens; TGF, transforming growth factor; TNF, tumor necrosis factor; TRP, transient receptor potential.

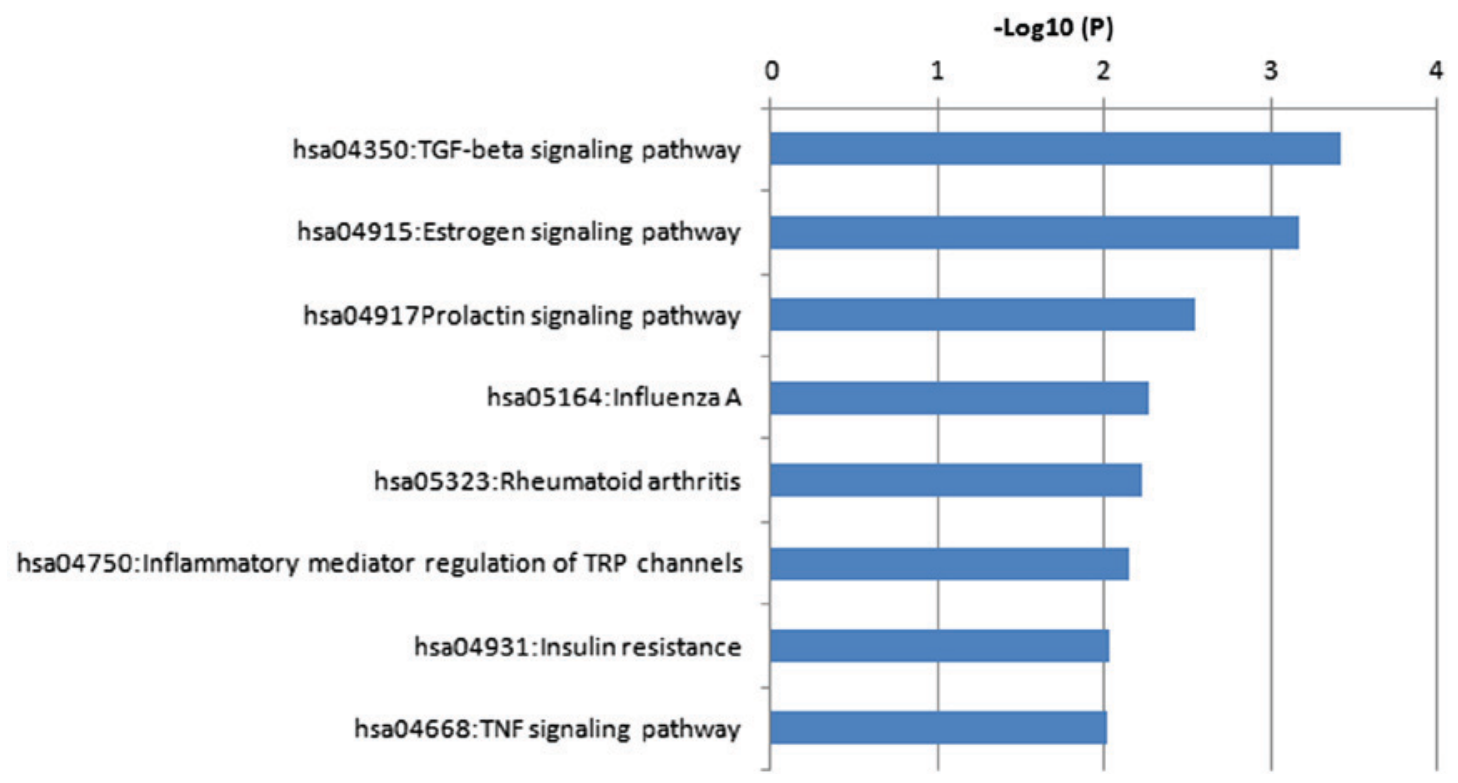

Figure 4. Kyoto Encyclopedia of Genes and Genomes pathway enrichment analysis. hsa, Homo sapiens; TGF, transforming growth factor; TNF, tumor necrosis factor; TRP, transient receptor potential.

or 'discs' with non-degenerated tissues. Among these studies, only one noted a similarity of genes involved in AF and NP degeneration by enrichment analysis and a PPI network, and to the best of our knowledge, no previous study has examined whether the degeneration of AF and NP proceeds via different pathophysiological processes at the cellular and the molecular level. Of note, Schubert et al (27) assessed AF and NP tissues from low-level degenerated disks and reported that 267 DEGs were upregulated in AF compared with NP tissues and that 52 DEGs were more highly expressed in NP compared with AF tissues. The authors further identified ankyrin repeat domain 29, adhesion G protein-coupled receptor L4, endomucin, LIM domain binding 2 and olfactomedin like $2 \mathrm{~A}$ as AF markers, and ArfGAP with RhoGAP domain, ankyrin repeat and $\mathrm{PH}$ domain 2 (ARAP2), cyclin dependent kinase inhibitor 2B (CDKN2B), defensin $\beta 1$, desmocollin 3 (DSC3) and erythroferrone as NP markers. Those identified as AF markers were upregulated in AF and those identified as NP markers were upregulated in NP. These results are consistent with those of the present study, which identified the upregulation of the NP markers ARAP2, CDKN2B and DSC3 in AF compared with those in NP tissues.

The present study uncovered several different pathophysiological aspects of AF and NP degeneration at the molecular level. These results enhance the current knowledge of the molecular mechanisms of IDD. The present analysis of $20 \mathrm{AF}$ and $20 \mathrm{NP}$ samples from the GSE70362 dataset retrieved from the GEO identified a total of 87 DEGs with $\mathrm{P}<0.05$ and $\mathrm{FC}>2$ in AF compared to NP samples including 48 upregulated and 39 downregulated DEGs. Enrichment analysis indicated that these DEGs were mainly involved in the GO terms of inflammatory response, ECM and RNA polymerase II transcription factor activity, as well as in the KEGG pathways of TGF- $\beta$ and estrogen signaling. KEGG pathway analysis revealed that MMP1 and IL6 were included in the genes enriched in rheumatoid arthritis, whereas BMP2 and THBS1 were among 


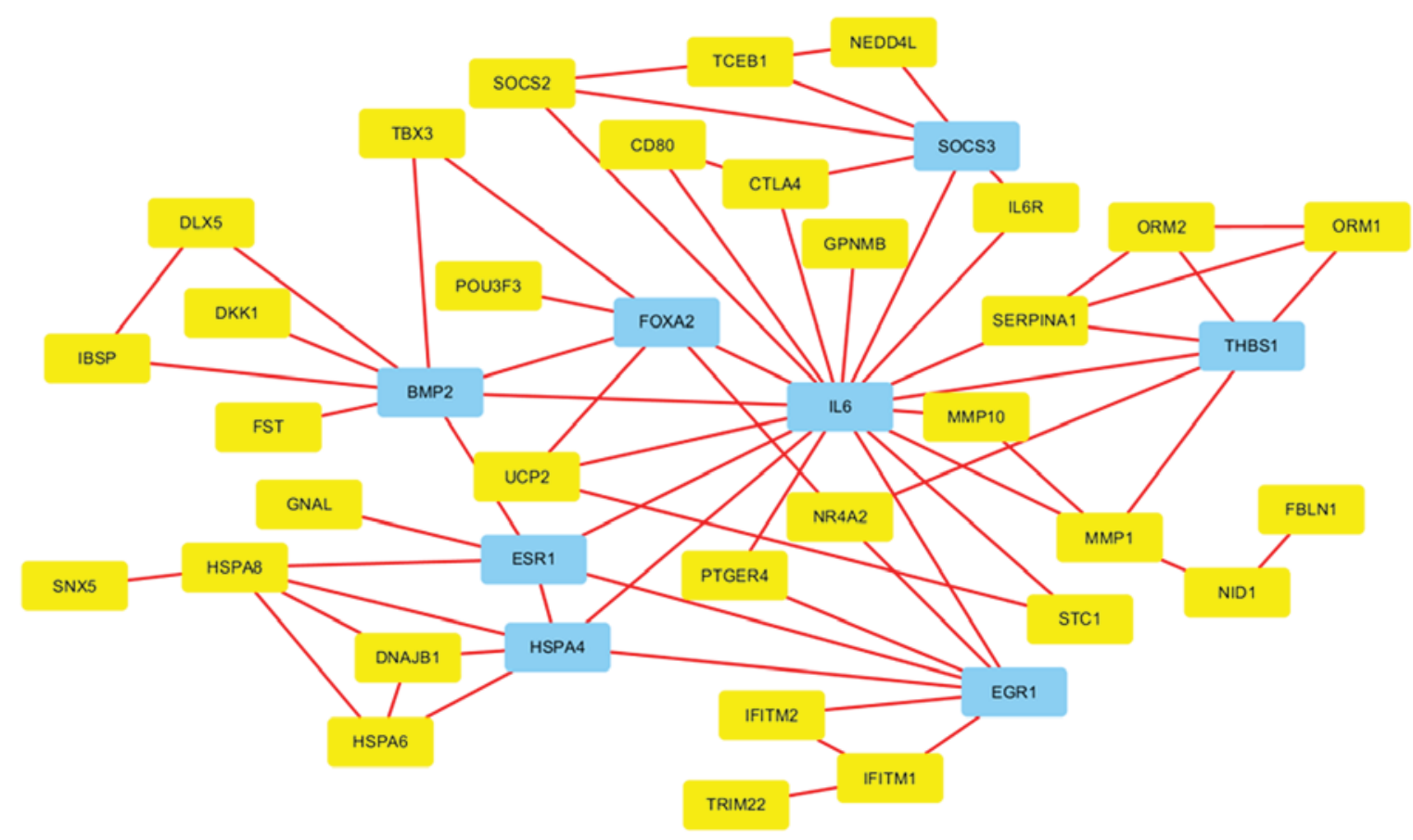

Figure 5. Protein-protein interaction network of differentially expressed genes. All nodes with a combined interaction score of $>0.4$ are displayed. The blue nodes have a central role with a degree of $>5.0$.

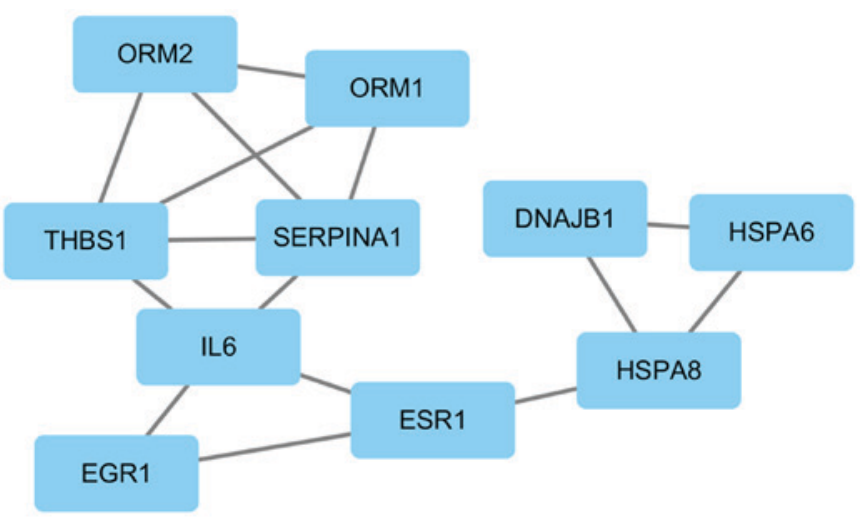

Figure 6. Significant modules were screened with the following criteria: Degree centrality cutoff, 2; node score cutoff, 0.2 ; k-core, 2; max depth, 100.

the genes enriched in the TGF- $\beta$ signaling pathway. In the PPI network, IL6 was identified as the central hub gene.

The expression of MMP1, which has been identified in $91 \%$ of IVDs, increases in degenerated IVDs (28). The significantly higher levels of MMP1 in degenerated IVDs compared with those in normal discs indicate a positive correlation between MMP1 and IDD (29). The expression of MMP1 was reported to be markedly associated with clefts and tears of AF and NP $(30,31)$. However, most of the abovementioned previous studies assessed 'disc tissues' or 'disc specimens' without differentiating between AF and NP. MMP1 is a type of collagenase with the characteristic function of cleaving interstitial collagens I, II and III $(32,33)$. MMP1 has the greatest degradation activity toward collagen III (34). Collagen III is the most common type of collagen in the inner AF, whereas the outer AF mainly consists of collagen I, and the NP mainly consists of collagen II (15-17,35). This pattern of collagen distribution and the different effects of MMP1 on the degradation of these collagens may explain for the upregulation of MMP1 in AF compared to NP observed in the present study.

BMP2 downregulates MMP13 and upregulates aggrecan, sex-determining region Y box 6 and type II collagen, and thus, it has an anti-catabolic effect on ECM enzymes in AF and NP (36). In NP and the inner AF, BMP2 increases aggrecan, as well as type I and II collagen, while simultaneously increasing proteoglycan synthesis and stimulating mitogenesis in the outer AF $(37,38)$. The biological effect of estrogen mainly occurs through ESR1 and -2 (39), with ESR2 as the predominant ESR (40). Basic research has confirmed that ESR1 is negatively correlated with the aggravation of NP degeneration (41). The present study revealed that the expression of ESR1 is upregulated in degenerated AF vs. NP; however, the detailed role of ESR1 in this context requires further investigation. The anti-angiogenic characteristics of THBS1 have long been known (42). A previous study reported strong immunoreactivity for THBS1 in the outer AF, suggesting that THBS1 may contribute to the avascular status of the IVD (43).

An inflammatory response is thought to initiate IDD, and pro-inflammatory molecules, including IL6, secreted by IVD cells are considered to mediate IDD (44). In addition, IL6 was identified as a hub gene in the PPI network that was constructed as part of the present study. Accordingly, it may be speculated that IL6 has a key role in IDD. This speculation is consistent with another study suggesting that IL6 is critical in IDD (45). Herniated IVDs have been reported to spontaneously produce IL6 (46). IL6 downregulates characteristic ECM proteins, including aggrecan and collagen II, in NP (47). This regulatory effect was specifically located to the site of herniation $(48,49)$. Shamji et al (50) detected higher IL6 expression in herniated IVD tissues than in non-degenerated autopsy samples, whereas Lee et al (51) identified no significant differences in IL6 
expression levels between degenerated IVDs and herniated NP. Most of these studies were based on herniated IVDs rather than on degenerated IVDs and did not distinguish between AF and NP specimens. None of these studies mentioned the difference in IL6 expression levels between AF and NP. In the present study, IL6 expression was upregulated with an FC of 2.27 in AF vs. NP tissues; the molecular biological implications of this observation require further investigation. With regard to the genes enriched in terms/pathways associated with the inflammatory response, except for the participation of IL6, BMP2, ESR1 and THBS1 in IDD, limited data are available to suggest the biological actions of F2R like trypsin receptor 1 , orosomucoid 1 and -2 , serpin family $\mathrm{F}$ member 1 , serpin family A member 1, SERPINA1, prostaglandin E receptor 4, phospholipase A2 group IVC and SOCS3 in IDD.

In conclusion, the present bioinformatics study revealed DEGs and their enriched functions/pathways in the AF and NP during IDD. A novel integrated understanding of certain molecular mechanisms of IDD was obtained. The different distribution patterns of collagen types and different degradation efficiencies of MMP1 on these collagen types may contribute to the upregulation of MMP1 in AF compared with that in NP. IL6 may be a key factor accounting for the different processes of degeneration in AF and NP. BMP2, ESR1 and THBS1 may also be involved in the different pathological changes observed in AF and NP. The present study is based on information available from a public database rather than from experimentation, and the biological implications of these different gene expression patterns require further investigation.

\section{Acknowledgements}

The authors gratefully acknowledge the writing assistance of Dr Zhirui Zhou (Department of Radiation Oncology, Fudan University Shanghai Cancer Center, Shanghai, China).

\section{Funding}

The present study was funded by the Key research and development projects of the Science \& Technology Department of Sichuan Province, China (grant no: 2018SZ0075).

\section{Availability of data and materials}

All data are already included in the article.

\section{Authors' contributions}

WY designed the study and, together with JL, performed data analysis and wrote the manuscript. DGG obtained funding and together with LSW advised on the study design and writing. MXY contributed to writing and English proofreading. DGG, LSW and MXY made substantial contributions to conception and design. All authors have given final approval of the version to be published.

\section{Ethics approval and consent to participate}

Not applicable.

\section{Patient consent for publication}

Not applicable.

\section{Competing interests}

The authors declare that they have no competing interests regarding this study.

\section{References}

1. Luoma K, Riihimäki H, Luukkonen $\mathrm{R}$, Raininko $\mathrm{R}$, Viikari-Juntura E and Lamminen A: Low back pain in relation to lumbar disc degeneration. Spine (Phila Pa 1976) 25: 487-492, 2000.

2. Andersson GB: Epidemiological features of chronic low-back pain. Lancet 354: 581-585, 1999.

3. Maniadakis $\mathrm{N}$ and Gray A: The economic burden of back pain in the UK. Pain 84: 95-103, 2000.

4. Katz JN: Lumbar disc disorders and low-back pain: Socioeconomic factors and consequences. J Bone Joint Surg Am 88 (Suppl 2): S21-S24, 2006.

5. Martin BI, Deyo RA, Mirza SK, Turner JA, Comstock BA, Hollingworth W and Sullivan SD: Expenditures and health status among adults with back and neck problems. JAMA 299: 656-664, 2008.

6. Yorimitsu E, Chiba K, Toyama Y and Hirabayashi K: Long-term outcomes of standard discectomy for lumbar disc herniation: A follow-up study of more than 10 years. Spine (Phila Pa 1976) 26: 652-657, 2001.

7. Li X, Han Y, Di Z, Cui J, Pan J, Yang M, Sun G, Tan J and Li L: Percutaneous endoscopic lumbar discectomy for lumbar disc herniation. J Clin Neurosci 33: 19-27, 2016.

8. Karasek $\mathrm{M}$ and Bogduk $\mathrm{N}$ : Twelve-month follow-up of a controlled trial of intradiscal thermal anuloplasty for back pain due to internal disc disruption. Spine (Phila Pa 1976) 25: 2601-2607, 2000.

9. Uschold TD, Fusco D, Germain R, Tumialan LM and Chang SW: Cervical and lumbar spinal arthroplasty: Clinical review. AJNR Am J Neuroradiol 33: 1631-1641, 2012.

10. Hilibrand AS and Robbins M: Adjacent segment degeneration and adjacent segment disease: The consequences of spinal fusion? Spine J 4 (6 Suppl): 190S-194S, 2004.

11. Kepler CK, Ponnappan RK, Tannoury CA, Risbud MV and Anderson DG: The molecular basis of intervertebral disc degeneration. Spine J 13: 318-330, 2013.

12. Martin MD, Boxell CM and Malone DG: Pathophysiology of lumbar disc degeneration: A review of the literature. Neurosurg Focus 13: E1, 2002.

13. Lyons G, Eisenstein SM and Sweet MB: Biochemical changes in intervertebral disc degeneration. Biochim Biophys Acta 673: 443-453, 1981.

14. Antoniou J, Steffen T, Nelson F, Winterbottom N, Hollander AP Poole RA, Aebi M and Alini M: The human lumbar intervertebral disc: Evidence for changes in the biosynthesis and denaturation of the extracellular matrix with growth, maturation, ageing, and degeneration. J Clin Invest 98: 996-1003, 1996.

15. Eyre DR and Muir H: Types I and II collagens in intervertebral disc. Interchanging radial distributions in annulus fibrosus. Biochem J 157: 267-270, 1976.

16. Roberts S, Menage J, Duance V and Wotton SF: Type III collagen in the intervertebral disc. Histochem J 23: 503-508, 1991.

17. Schollmeier G, Lahr-Eigen $\mathrm{R}$ and Lewandrowski KU: Observations on fiber-forming collagens in the anulus fibrosus. Spine (Phila Pa 1976) 25: 2736-2741, 2000.

18. Kazezian Z, Gawri R, Haglund L, Ouellet J, Mwale F, Tarrant F, O'Gaora P, Pandit A, Alini M and Grad S: Gene expression profiling identifies interferon signalling molecules and IGFBP3 in human degenerative annulus fibrosus. Sci Rep 5: 15662, 2015.

19. Thompson JP, Pearce RH, Schechter MT, Adams ME, Tsang IK and Bishop PB: Preliminary evaluation of a scheme for grading the gross morphology of the human intervertebral disc. Spine (Phila Pa 1976) 15: 411-415, 1990.

20. Gruber HE, Hoelscher GL, Ingram JA and Hanley EN Jr: Genome-wide analysis of pain-, nerve- and neurotrophin-related gene expression in the degenerating human annulus. Mol Pain 8: 63, 2012 . 
21. Gruber HE, Ingram JA, Hoelscher GL, Zinchenko N, Hanley EN $\mathrm{Jr}$ and Sun Y: Asporin, a susceptibility gene in osteoarthritis, is expressed at higher levels in the more degenerate human intervertebral disc. Arthritis Res Ther 11: R47, 2009.

22. Tsai TT, Lai PL, Liao JC, Fu TS, Niu CC, Chen LH, Lee MS, Chen WJ and Fang HC: Increased periostin gene expression in degenerative intervertebral disc cells. Spine J 13: 289-298, 2013.

23. Gruber HE, Hoelscher GL and Hanley EN Jr: Annulus cells from more degenerated human discs show modified gene expression in $3 \mathrm{D}$ culture compared with expression in cells from healthier discs. Spine J 10: 721-727, 2010.

24. He J, Xue R, Li S, Lv J, Zhang Y, Fan L, Teng Y and Wei H: Identification of the potential molecular targets for human intervertebral disc degeneration based on bioinformatic methods. Int J Mol Med 36: 1593-1600, 2015.

25. Tang Y, Wang S, Liu Y and Wang X: Microarray analysis of genes and gene functions in disc degeneration. Exp Ther Med 7 343-348, 2014

26. Guo W, Zhang B, Li Y, Duan HQ, Sun C, Xu YQ and Feng SQ Gene expression profile identifies potential biomarkers for human intervertebral disc degeneration. Mol Med 16: 8665-8672, 2017.

27. Schubert AK, Smink JJ, Arp M, Ringe J, Hegewald AA and Sittinger M: Quality assessment of surgical disc samples discriminates human annulus fibrosus and nucleus pulposus on tissue and molecular level. Int J Mol Sci 19: pii: E1761, 2018.

28. Roberts S, Caterson B, Menage J, Evans EH, Jaffray DC and Eisenstein SM: Matrix metalloproteinases and aggrecanase: Their role in disorders of the human intervertebral disc. Spine (Phila Pa 1976) 25: 3005-3013, 2000.

29. Deng B, Ren JZ, Meng XQ, Pang CG, Duan GQ, Zhang JX Zou H, Yang HZ and Ji JJ: Expression profiles of MMP-1 and TIMP-1 in lumbar intervertebral disc degeneration. Genet Mol Res 14: 19080-19086, 2015.

30. Weiler C, Nerlich AG, Zipperer J, Bachmeier BE and Boos N: 2002 SSE Award Competition in Basic Science: Expression of major matrix metalloproteinases is associated with intervertebral disc degradation and resorption. Eur Spine J 11: 308-320, 2002.

31. Le Maitre CL, Freemont AJ and Hoyland JA: Localization of degradative enzymes and their inhibitors in the degenerate human intervertebral disc. J Pathol 204: 47-54, 2004.

32. Matsui Y, Maeda M, Nakagami W and Iwata $H$ : The involvement of matrix metalloproteinases and inflammation in lumbar disc herniation. Spine (Phila Pa 1976) 23: 863-869, 1998.

33. Visse R and Nagase H: Matrix metalloproteinases and tissue inhibitors of metalloproteinases: Structure, function, and biochemistry. Circ Res 92: 827-839, 2003.

34. Welgus HG, Jeffrey JJ and Eisen AZ: The collagen substrate specificity of human skin fibroblast collagenase. J Biol Chem 256 9511-9515, 1981.

35. Roberts S, Menage J, Duance V, Wotton S and Ayad S: 1991 Volvo Award in basic sciences. Collagen types around the cells of the intervertebral disc and cartilage end plate: An immunolocalization study. Spine (Phila Pa 1976) 16: 1030-1038, 1991.

36. Ye S, Ju B, Wang H and Lee KB: Bone morphogenetic protein-2 provokes interleukin-18-induced human intervertebral disc degeneration. Bone Joint Res 5: 412-418, 2016.

37. Kim DJ, Moon SH, Kim H, Kwon UH, Park MS, Han KJ, Hahn SB and Lee HM: Bone morphogenetic protein-2 facilitates expression of chondrogenic, not osteogenic, phenotype of human intervertebral disc cells. Spine (Phila Pa 1976) 28: 2679-2684, 2003.
38. Kim H, Lee JU, Moon SH, Kim HC, Kwon UH, Seol NH, Kim HJ, Park JO, Chun HJ, Kwon IK and Lee HM: Zonal responsiveness of the human intervertebral disc to bone morphogenetic protein-2. Spine (Phila Pa 1976) 34: 1834-1838, 2009.

39. Hammes SR and Levin ER: Extranuclear steroid receptors: Nature and actions. Endocr Rev 28: 726-741, 2007.

40. Bay-Jensen AC, Slagboom E, Chen-An P, Alexandersen P, Qvist P, Christiansen C, Meulenbelt I and Karsdal MA: Role of hormones in cartilage and joint metabolism: Understanding an unhealthy metabolic phenotype in osteoarthritis. Menopause 20: 578-586, 2013

41. Song XX, Yu YJ, Li XF, Liu ZD, Yu BW and Guo Z: Estrogen receptor expression in lumbar intervertebral disc of the elderly: Gender- and degeneration degree-related variations. Joint Bone Spine 81: 250-253, 2014.

42. Chen H, Herndon ME and Lawler J: The cell biology of thrombospondin-1. Matrix Biol 19: 597-614, 2000.

43. Gruber HE, Ingram JA and Hanley EN Jr: Immunolocalization of thrombospondin in the human and sand rat intervertebral disc. Spine (Phila Pa 1976) 31: 2556-2561, 2006.

44. Risbud MV and Shapiro IM: Role of cytokines in intervertebral disc degeneration: Pain and disc content. Nat Rev Rheumatol 10: 44-56, 2014.

45. Dagistan Y, Cukur S, Dagistan E and Gezici AR: Importance of IL-6, MMP-1, IGF-1, and BAX levels in lumbar herniated disks and posterior longitudinal ligament in patients with sciatic pain. World Neurosurg 84: 1739-1746, 2015.

46. Kang JD, Georgescu HI, McIntyre-Larkin L, Stefanovic-Racic M, Donaldson WF III and Evans CH: Herniated lumbar intervertebral discs spontaneously produce matrix metalloproteinases, nitric oxide, interleukin-6, and prostaglandin E2. Spine (Phila Pa 1976) 21: 271-277, 1996

47. Studer RK, Vo N, Sowa G, Ondeck C and Kang J: Human nucleus pulposus cells react to IL-6: Independent actions and amplification of response to IL-1 and TNF- $\alpha$. Spine (Phila Pa 1976) 36: 593-599, 2011.

48. Takahashi H, Suguro T, Okazima Y, Motegi M, Okada Y and Kakiuchi T: Inflammatory cytokines in the herniated disc of the lumbar spine. Spine (Phila Pa 1976) 21: 218-224, 1996.

49. Specchia N, Pagnotta A, Toesca A and Greco F: Cytokines and growth factors in the protruded intervertebral disc of the lumbar spine. Eur Spine J 11: 145-151, 2002.

50. Shamji MF, Setton LA, Jarvis W, So S, Chen J, Jing L, Bullock R, Isaacs RE, Brown C and Richardson WJ: Proinflammatory cytokine expression profile in degenerated and herniated human intervertebral disc tissues. Arthritis Rheum 62: 1974-1982, 2010.

51. Lee S, Moon CS, Sul D, Lee J, Bae M, Hong Y, Lee M, Choi S, Derby R, Kim BJ, et al: Comparison of growth factor and cytokine expression in patients with degenerated disc disease and herniated nucleus pulposus. Clin Biochem 42: 1504-1511, 2009.

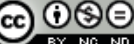

This work is licensed under a Creative Commons Attribution-NonCommercial-NoDerivatives 4.0 International (CC BY-NC-ND 4.0) License. 